\title{
A PROBLEM IN ADDITIVE NUMBER THEORY
}

\author{
DONALD QUIRING
}

ABSTRACT. For every real number $\alpha, 0<\alpha<1$, a sequence $A=\left\{a_{1}, a_{2}, \cdots\right\}$ is constructed for which the density of $A$ is $\alpha$ and $A$ has the following property: Given any $n$ distinct positive integers $\left\{b_{1}, b_{2}, \cdots, b_{n}\right\}$ the sequence consisting of all numbers of the form $a_{i}+b_{j}$ has density $1-(1-\alpha)^{n}$.

Let $A=\left\{a_{1}, a_{2}, \cdots\right\}$ and $B=\left\{b_{1}, b_{2}, \cdots\right\}$ be increasing sequences of positive integers. The sequence $A+B$ is defined as the increasing sequence consisting of all the sums $a_{i}+b_{j}$. Let $A(n)$ be the number of elements of $A$ that are less than $n$. The limit $A(n) / n$, if it exists, is called the density of $A$ and designated $d(A)$.

P. Erdös and A. Renyi [1] have shown that for every $\alpha, 0<\alpha<1$, there exists a sequence $A$ of density $\alpha$ which has the property that for any infinite sequence $B, d\left(A+\left\{b_{1}, \cdots, b_{n}\right\}\right)=1-(1-\alpha)^{n}$. This implies $d(A+B)=1$. The purpose of this paper is to provide examples of such sequences.

If $\alpha$ is rational we proceed as follows. Express $\alpha$ as a quotient of natural numbers, $\alpha=p / q, q>p$. List all the natural numbers in order in base $q$ notation to obtain a sequence

$$
S=\left\{s_{1}, s_{2}, \cdots\right\}, \quad 0 \leqq s_{i} \leqq q-1 .
$$

Define $A$ by $A=\left\{i \mid 0 \leqq s_{i} \leqq p-1\right\}$. Then $d(A)=\alpha$ and if $B$ is an increasing sequence of positive integers $d\left(A+\left\{b_{1}, \cdots, b_{n}\right\}\right)=1-(1-\alpha)^{n}$.

We prove that $A$ has these properties in the case $\alpha=1 / 2$. The other cases can be handled by essentially the same method.

List the natural numbers in order in base 2 notation separated by hyphens as follows:

$$
\text { 1-10-11-100-101-110-111- - . }
$$

We treat this list as a sequence of digits $s_{1}, s_{2}, \cdots$ with hyphens between $s_{1}$ and $s_{2}, s_{3}$ and $s_{4}$, etc. Define $\left\{\left(s_{i}, t_{i}, u_{i}\right)\right\}$ by letting $s_{i}=0$ or 1 be the $i$ th entry in the above sequence $t_{i}=\inf _{j}$ (there is one hyphen between the $i$ th entry and the $i-j$ th entry), and $u_{i}=\inf _{j}$ (there is one hyphen between the $i$ th entry and the $i+j$ th entry).

Received by the editors July 5, 1972 and, in revised form, August 1, 1972. AMS (MOS) subject classifications (1970). Primary 10L05, 10L10. 
Let $A=\left\{i \mid s_{i}=0\right\}$. We show that $d\left(A+\left\{b_{1}, \cdots, b_{n}\right\}\right)=1-2^{-n}$ for an arbitrary increasing sequence $B$. The case $n=1$ will then give us $d(A)=1 / 2$.

Define sequences $T_{n}$ and $T_{n}^{k}$ by

$$
T_{n}=\left\{i \mid t_{i} \geqq b_{n}+2\right\} \quad \text { and } \quad T_{n}^{k}=\left\{i \mid t_{i} \geqq b_{n}+2, u_{i}=k\right\} .
$$

Let $i_{m}=\inf _{i}\left(u_{i}=m+1\right)$; that is,

$$
i_{m}=1+\sum_{k=1}^{m} k 2^{k-1}=(m-1) 2^{m}+2 .
$$

Define a sequence $C_{n}$ as the intersection of $T_{n}$ with the complement of $A+\left\{b_{1}, \cdots, b_{n}\right\}$. Since $d\left(T_{n}\right)$ is clearly equal to 1 it suffices to show $d\left(C_{n}\right)=2^{-n}$. Note that $C_{n}=\left\{i \mid i \in T_{n}\right.$ and $\left.s_{i-b_{1}}=s_{i-b_{2}}=\cdots=s_{i-b_{n}}=1\right\}$ and that for $i_{m} \leqq i<i_{m+1}, T_{n}(i)=\sum_{k=1}^{m-b}{ }_{n}^{-1} T_{n}^{k}(i) . T_{n}(i)$ is the number of elements of $T_{n}$ that are less than $i$.

Among any $2^{b_{n}+k}$ consecutive elements of $T_{n}^{k}$ there are $2^{b_{n}+k-n}$ elements of $C_{n}$. This is because among any $2^{b_{n}+k}$ consecutive natural numbers every possible combination of the last $b_{n}+k$ digits appears exactly once.

Therefore, for all $i$,

$$
\left(C_{n} \cap T_{n}^{k}\right)(i)-2^{b_{n+k-n}} \leqq 2^{-n} T_{n}^{k}(i) \leqq\left(C_{n} \cap T_{n}^{k}\right)(i)+2^{b_{n+k-n}},
$$

and for $i_{m} \leqq i<i_{m+1}, m>b_{n}$,

$$
C_{n}(i)-\sum_{k=1}^{m-b n-1} 2^{b_{n+k-n}} \leqq 2^{-n} T_{n}(i) \leqq C_{n}(i)+\sum_{k=1}^{m-b_{n-1}} 2^{b_{n}+k-n}
$$

So $C_{n}(i)-2^{m-n}<2^{-n} T_{n}(i)<C_{n}(i)+2^{m-n}$ and $\left|C_{n}(i) / i-2^{-n} T_{n}(i) / i\right|<2^{m-n} / i$. Since we are assuming $i \geqq i_{m}>(m-1) 2^{m}$, we have $\left|C_{n}(i)\right| i-2^{-n} T_{n}(i)|i|<$ $2^{-n} /(m-1)$. We know that $\lim _{i \cdot \infty} T_{n}(i) / i=d\left(T_{n}\right)=1$ and it follows that $\lim _{i \rightarrow \infty}\left|C_{n}(i) / i-2^{-n}\right| \leqq 2^{-n} /(m-1)$ for all $m$. We conclude that $d\left(C_{n}\right)=2^{-n}$ and the proof is complete.

We turn now to the case where $\alpha$ is not rational. Express $\alpha$ as a limit of rational numbers $\alpha_{j}, \alpha=\lim \alpha_{j}$, and let $A_{\alpha_{j}}$ be the sequence with density $\alpha_{j}$ constructed above. Compose $A_{\alpha}$ of increasingly long segments of the sequences $A_{\alpha,}$ as follows.

Define inductively integers $N_{j}$ and finite sequences $E_{j}$ by

$$
E_{j}=\left(A_{\alpha_{1}} \cap\left(1, N_{1}\right)\right) \cap\left(A_{\alpha_{2}} \cap\left(N_{1}+1, N_{2}\right)\right) \cap \cdots
$$

choosing $N_{j}$ large enough that

$$
\cap\left(A_{\alpha_{j}} \cap\left(N_{j-1}+1, N_{j}\right)\right)
$$

$$
\sup _{C}\left|\left(E_{j}+C\right)\left(N_{j}\right) / N_{j}-\left(1-\left(1-\alpha_{j}\right)^{n}\right)\right|<1 / j
$$


where the supremum is taken over all subsets $C$ of $\{1,2, \cdots, j\}$ and $n$ is the number of elements of $C$. If we let $A_{\alpha}=\bigcup E_{j}$ then $A_{\alpha}$ clearly has the desired property.

\section{REFERENCE}

1. P. Erdös and A. Renyi, On some applications of probability methods to additive number theoretic problems, Contributions to Ergodic Theory and Probability (Proc. Conf., Ohio State Univ., Columbus, Ohio, 1970), Springer, Berlin, 1970, pp. 37-44. MR 43 \#1938.

Department of Mathematics, University of New Mexico, Albuquerque, New MeXICO 87106

Current address: Department of Mathematics, Louisiana State University, New Orleans, Louisiana 70122 